\title{
Comportamiento clínico-epidemiológico de la influenza en niños hospitalizados
}

\author{
LUIS DELPIANO M. ${ }^{1}$, BYRON GUILLEN B. ${ }^{2}$ y M. CRISTINA CASADO F. ${ }^{2}$
}

\section{Clinical and epidemiological pattern of influenza in pediatric inpatients}

The infection due to influenza virus occurs in seasonal outbreaks and has a severe clinical course in high risk population. This prospective study was done to evaluate the epidemiological and clinical behavior of influenza virus in children admitted during the 1999 outbreak to the San Borja Arriarán hospital. We included 175 children (60\% younger than 12 months) admitted for acute respiratory infection in which influenza virus was found by IFD during the period from May to September 1999. Influenza A was detected in $95 \%$ of cases. Group 1 included 46 patients with risk factors and group 2 , 129 healthy children. Fifty-one percent of the whole group presented complications. Group 1 showed a significant increase in nosocomial acquisition of influenza virus infection, as well as total days of oxygen supplementation, of intensive care unit stay and total hospitalization $(\mathrm{p}<0.05)$. We recognize a high risk group of children, in which we should focus preventive programs such as vaccines and minimizing nosocomial infections.

Key words: Influenza; Acute respiratory infection; Nosocomial infections.

\section{Introducción}

La infección por virus influenza ocurre de manera estacional en climas temperados. La importancia de este virus radica en su capacidad de generar usualmente epidemias de severidad variable y diseminaciones globales ampliamente difundidas -pandemias- que ocurren a intervalos irregulares impredecibles, siendo en estos períodos cuando la morbi-mortalidad relacionada con este patógeno se incrementa dramáticamente ${ }^{1}$. El carácter antigénico variable e impredecible ubica a este agente en un lugar preponderante entre las infecciones emergentes y reemergentes ${ }^{2}$.

La influenza se caracteriza por la súbita aparición de signos y síntomas como fiebre $\left(38-40^{\circ} \mathrm{C}\right)$, mialgias, cefalea, calofríos, decaimiento, tos no productiva, faringitis y rinitis ${ }^{3}$. En niños, además puede producir síntomas gastrointestinales: vómitos, dolor abdominal, diarrea y desencadenar convulsiones febriles. En población de riesgo (enfermedad pulmonar crónica, cardíaca o neurológica) esta infección puede exacerbar su condición de base ${ }^{1}$. Las complicaciones en el tracto respiratorio inferior incluyen deterioro de enfermedad obstructiva crónica, crisis asmáticas, laringitis, bronquitis, bronquiolitis, y neumonías virales primarias o con sobreinfección bacteriana ${ }^{4}$.

De forma característica, los brotes epidémicos por virus influenza ocurren durante los meses de otoño e invierno y son responsables de aproximadamente 20.000 muertes por año en los Estados Unidos de Norteamérica ${ }^{5-6}$. La morbimortalidad asociada a esta patología afecta a todos los grupos etarios ${ }^{2}$ y es reconocido como agente de brotes de infección intrahospitalaria en lactantes y niños de salas pediátricas generales ${ }^{6-7}$, y también en unidades cerradas como las de cuidado intensivo neonatal ${ }^{8}$. Los niños son fuente importante de diseminación viral en la comunidad dado el contacto estrecho que existe en los lugares destinados para su cuidado, con una alta tasa de incidencia, que en época de brote alcanza el $40 \%$ en la población infantil; esta cifra representa el doble de la incidencia objetivada en otros grupos como adultos jóvenes y personas de la tercera

Hospital Clínico San Borja Arriarán:

Unidad de Aislamiento Infecciosos. Servicio de Pediatría.

2 Residente Servicio de Pediatría. 
edad $^{9}$ La tasa de mortalidad en niños es mucho menor que en senescentes ${ }^{10}$.

En muchos países existe vigilancia epidemiológica de influenza, pero a pesar de estos esfuerzos, la cuantificación real de la morbilidad causada por este virus es complicada por la pobre especificidad del diagnóstico clínico (muchos agentes infecciosos producen sintomatología similar), y por la diversidad de definiciones clínicas usadas para reportar los casos de influenza en diferentes estudios de supervigilancia, generalmente también imprecisas ${ }^{11-13}$.

Se dispone de vacuna contra virus influenza, pero sólo está indicada sobre los 6 meses de edad, y en personas con una alta probabilidad de experimentar complicaciones, es el caso de las personas sobre 65 años de edad y los portadores de patología crónica. El beneficio de la vacunación sólo se observará si la vacuna contiene la cepa viral circulante. Sin embargo, se han reportado buenos resultados cuando se ha utilizado en niños sanos reduciendo la incidencia de otitis media aguda y la prescripción de antimicrobianos $^{14-15}$.

En una revisión de los casos de influenza hospitalizados en el Servicio de Pediatría del Hospital Clínico San Borja Arriarán (HCSBA) durante 1998, se estudió la evolución de 27 niños, destacando la falta de vacunación en pacientes portadores de patología crónica, quienes además presentaron un mayor número de complicaciones ${ }^{16}$.

Esta observación sugirió la hipótesis que los pacientes hospitalizados en Pediatría del HCSBA y portadores de una condición preexistente o factores de riesgo (respiratorios, neurológicos, cardiológicos o prematurez), tienen una mayor posibilidad de presentar complicaciones en el curso de una influenza, que los pacientes sin morbilidad significativa previa. Se pretendió entonces describir el comportamiento clínico de niños hospitalizados con influenza y comparar la evolución entre dos grupos de diferente riesgo.

\section{Pacientes y Método}

Se diseñó un estudio prospectivo descriptivo, clínico-epidemiológico, en el que se incluyó a los pacientes internados en el Servicio de Pediatría del HCSBA durante el período de otoño-invierno de 1999, con diagnóstico de infección respiratoria aguda (IRA). La etiología fue confirmada por el método de IFD, con panel que incluye detección de VRS, virus parainfluenza (paraFlu), ADV, virus influenza A (FluA) y virus influenza B (FluB), en muestra de aspirado nasofaríngeo to- madas por enfermeras o kinesiólogos y transportadas al laboratorio de Inmunología del hospital en tubos "T" con PBS (solución buffer - fosfato con $\mathrm{pH} 7,2$ ) manteniendo una rigurosa cadena de frío.

En el laboratorio, luego de homogenizar la muestra se la centrifugó durante $8 \mathrm{~min}$ a 1.500 rpm a una temperatura de $4^{\circ} \mathrm{C}$ por dos veces. Se realizaron tres placas por cada muestra en estudio, que se dejaron secar a temperatura ambiente y fijaron con acetona fría. Posteriormente, se tiñeron utilizando el reactivo DFA (The Light Diagnostics Respiratory panel 1 DFA-Chemicon International, INC). Incubada la muestra a $37^{\circ} \mathrm{C}$ en cámara húmeda, se lavó dos veces con PBS, secó y agregó una muestra de glicerina tamponada, para ser observada al microscopio de inmunofluorescencia.

A cada paciente con IFD positiva para virus influenza (se excluyó pacientes con asociación de influenza a otros virus), se le completó un formulario de seguimiento llenado por los investigadores de manera cronológica de acuerdo al orden de ingreso de los pacientes; en este formulario se consignaron las siguientes variables:

- Epidemiológicas: edad, sexo, contactos intrafamiliares o contagio intrahospitalario, uso de vacuna antigripal, días previos de enfermedad.

- Clínicas: síntomas y signos previo a ingreso, temperatura de ingreso, morbilidad preexistente.

- Laboratorio: variables hematológicas: hemograma, recuento y fórmula diferencial de leucocitos, PCR cuantitativa, radiografía de tórax.

- Evolutivas: días de hospitalización, días de oxigenoterapia, uso de antimicrobianos, complicaciones, derivación a unidad de cuidados intensivos pediátricos.

Los pacientes que ingresaron al estudio se dividieron para el análisis en dos grupos de acuerdo a sus antecedentes. El Grupo 1, correspondió a niños con infección aguda por influenza pero con alguna de las siguientes condiciones preexistentes: antecedente de prematurez, daño neurológico, daño pulmonar o cardiopatía. El Grupo 2, correspondió a los pacientes con infección aguda por influenza pero que carecieron de antecedentes mórbidos significativos.

\section{Análisis estadístico}

Se aplicó test de significación estadística por medio del método de $\chi^{2}$, para muestras no paramétricas, teniendo como variable dependiente a 
la infección por influenza y como variable independiente a las complicaciones. Para la determinación de diferencias entre las complicaciones presentadas de acuerdo al grupo se utilizó la diferencia de porcentajes, y para objetivar la diferencia de promedios entre variables como días de oxigenoterapia y días de hospitalización se utilizó t de student. Todas estas pruebas se realizaron considerando un error alfa con $\mathrm{p}<0,05$.

\section{Resultados}

En el período comprendido entre mayo y agosto de 1999 ingresaron al Servicio de Pediatría del HCSBA un total de 1.548 niños, planteándose sospecha de infección respiratoria aguda viral en 1.300 pacientes $(84 \%)$, confirmándose un agente etiológico viral en 733 niños (56\%). Se documentó la influenza en 175 pacientes, $11,3 \%$ del total de ingresos en el servicio durante este período.

La distribución etiológica viral de los pacientes hospitalizados en este período, se presenta de acuerdo a su aparición en semanas epidemiológicas, en la Figura 1.

El virus influenza apareció desde la semana 18 , en rango ascendente hasta la semana 22 (nivel máximo) y se prolongó con alguna variación hasta la semana 32, con una duración de 14 semanas. Durante este período se sobrepuso el brote anual de infección por VRS, observándose el fenómeno de coexistencia viral. Los casos y distintas asociaciones de coexistencia viral diagnosticados por IFD se presentan en la Tabla 1.

Se confirmó la influenza en 175 pacientes, con un claro predominio de FluA (95\%) sobre FluB (5\%). El total de pacientes con influenza fue clasificado en dos grupos, ingresando 46 pacientes al grupo 1 y 129 al grupo 2 .

Sesenta y uno por ciento de los niños (107) tenía menos de 12 meses de edad y 32\% (56) menos de 6 meses. La edad promedio de los niños fue 14 meses $\pm 17,1$ con una mediana de 9 meses (Figura 2). No se encontró diferencias en la proporción entre niños y niñas 1:1, destacando que en $10 \%$ de los pacientes existió el antecedente epidemiológico de al menos un contacto intrafamiliar con síntomas respiratorios al momento de su ingreso.

Las edades fueron para el Grupo 1 de 22,5 meses promedio $(0,7-168)$ con una mediana de 14 y para el Grupo 2 de 12,2 meses promedio (0,5-96) con una mediana de 8 meses.

En los 175 niños evaluados, los síntomas previos a la hospitalización aparecieron 4,1 $\pm 2,9$ días antes de ingresar. El principal motivo de consulta fue fiebre $>38,5^{\circ} \mathrm{C}$, presente en $47 \%$ de los pacientes al momento del ingreso. Se observó síndrome diarreico agudo (SDA) concomitante con los síntomas respiratorios al inicio del cuadro, en 6 pacientes y convulsión febril en 3 . Entre las variables hematológicas destacó el recuento de leucocitos promedio al ingreso de $12.347 \pm 6.157 / \mathrm{mm}^{3}$, con desviación izquierda $>500$ baciliformes como recuento absoluto en el $54 \%$ y PCR promedio $24,4 \pm 20 \mathrm{mg} / \mathrm{l}$ (VN: 10 $\mathrm{mg} / \mathrm{l})$. Para la obtención de estos datos, se excluyó a los pacientes que tuvieron una infección bacteriana concomitante como ITU (3 pacientes), meningitis aguda (1) y empiema pleural (2).

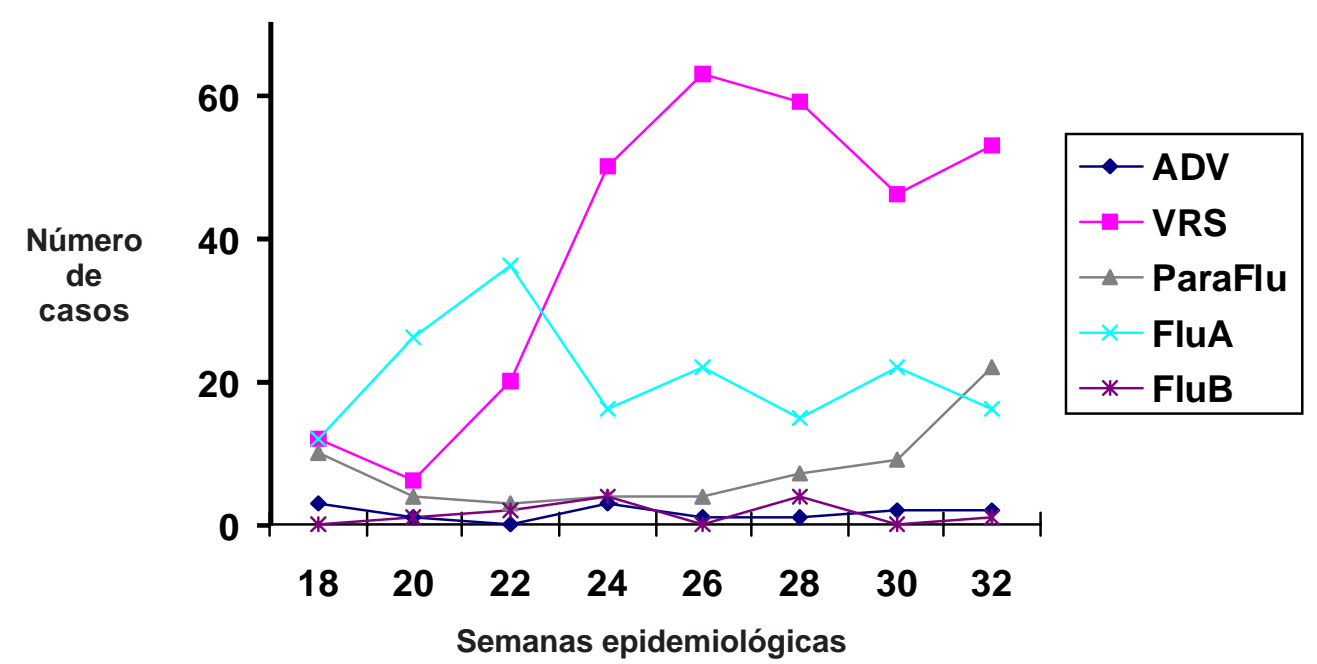

Figura 1. Distribución etiológica de casos confirmados de infección respiratoria viral en niños hospitalizados en el Servicio de Pediatría del HCSBA. Mayo - agosto de 1999. $(\mathrm{n}=733)$. 
Tabla 1. Coinfecciones virales obtenidas por IFD en niños hospitalizados en el Servicio de Pediatría del HCSBA. Mayo-agosto 1999

\begin{tabular}{|c|c|c|c|c|c|}
\hline \multirow[t]{2}{*}{ Virus } & \multicolumn{4}{|c|}{ Meses } & \multirow{2}{*}{$\begin{array}{c}\text { Total } \\
\mathbf{N}^{\circ}\end{array}$} \\
\hline & Mayo & Junio & Julio & Agosto & \\
\hline VRS-FluA & 4 & 17 & 47 & 35 & 103 \\
\hline ParaFlu-FluA & - & 1 & 5 & 7 & 13 \\
\hline ADV-VRS & - & 5 & 2 & 1 & 8 \\
\hline VRS- ParaFlu & - & 3 & 7 & 3 & 13 \\
\hline
\end{tabular}

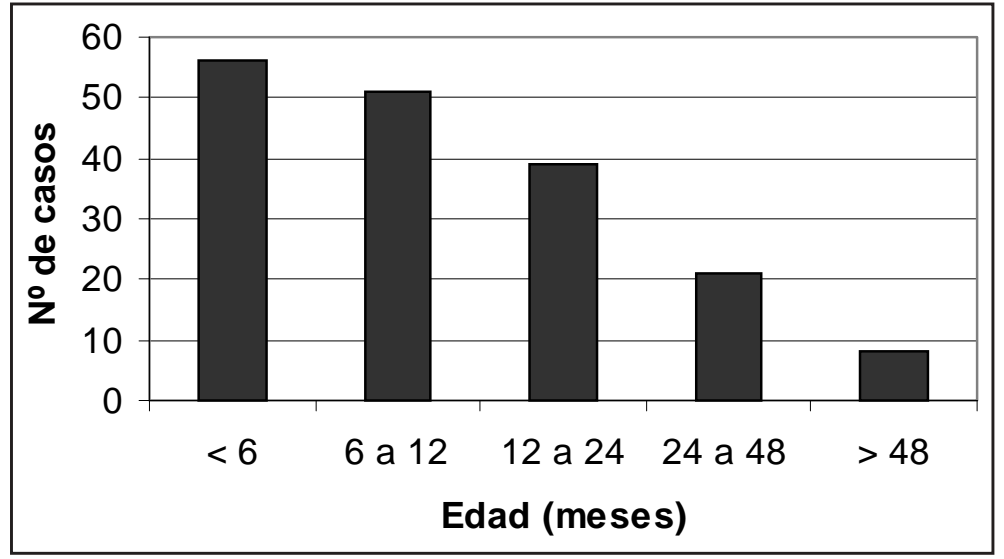

Figura 2. Distribución por edad de pacientes con influenza, mayo-agosto de 1999. Servicio de Pediatría HCSBA $(n=175)$.
$\mathrm{Al}$ ingreso se tomó radiografía de tórax en 171 niños $(97,7 \%)$, en 9 pacientes $(5,5 \%)$ fue informada como normal. Se observó un patrón radiológico único en 55 pacientes (32\%), distribuido en consolidaciones neumónicas en 27 pacientes $(15,7 \%)$, infiltrado intersticial en 25 pacientes (14\%) e hiperinsuflación en 3 (1,7\%). Un patrón radiológico mixto se presentó en 107 pacientes (61\%). Las atelectasias se objetivaron en $11 \%$ de las radiografías de ingreso.

En 51\% de los pacientes evaluados se observaron complicaciones, 27 niños $(56,5 \%)$ pertenecientes al Grupo 1 y 61 (47,2 \%) en el Grupo 2. No existió diferencias en el tipo de complicaciones entre los dos grupos. Los pacientes de ambos grupos, independientemente de sus antecedentes, fueron susceptibles de complicarse, sin existir diferencia en la prueba del $\chi^{2}$ con $p=0,007$.

Las principales complicaciones registradas durante la evolución de los pacientes se detallan en la Tabla 2 destacando:

- Respiratorias: la sobreinfección bacteriana planteada en 41 pacientes $(23,4 \%)$ por persistencia de fiebre y viraje en los parámetros hematológicos (leucocitosis y aumento de PCR sobre $80 \mathrm{mg} / \mathrm{l}$ ), sólo se pudo confirmar por hemocultivos en 2 pacientes, en los que se aisló Streptococcus pneumoniae susceptible a penicilina.
Veintinueve pacientes $(16,5 \%)$ evolucionaron con atelectasias en las radiografías de tórax de control, 13 de ellos pertenecientes al Grupo 1 y 16 al Grupo 2. Presentaron síndrome bronquial obstructivo (SBO) moderado a severo (score de tal > 6) el $15 \%$ de los niños.

El desarrollo de insuficiencia respiratoria fue la principal causa de derivación a la UCI. Siete pacientes $(4 \%)$ requirieron apoyo con ventilación mecánica, 5 pacientes en el Grupo 1 y 2 pacientes en el Grupo 2.

Apnea, laringitis y neumomediastino se presentaron cada uno en 2 pacientes; esta última complicación se presentó en pacientes sin ventilación mecánica, uno con laringitis y otro con un SBO severo.

OMA se diagnosticó por otoscopia durante la hospitalización en 4 pacientes $(2,2 \%)$ mientras que sinusitis aguda y conjuntivitis purulenta en 1 solo caso $(0,57 \%)$.

- Cardíacas: miocarditis con alteraciones de enzimas y electrocardiograma, en 2 niños $(1,1 \%)$.

- Neurológicas: convulsión febril en 3 niños $(1,7 \%)$

- Digestivas: seis pacientes $(3,4 \%)$ desarrollaron SDA, todos ellos debidas a infección intrahospitalaria por rotavirus, cinco de los cuales pertenecían al Grupo 2. 
Tabla 2. Tipo de complicación distribuidas por grupo de niños con infección por virus influenza. Servicio de Pediatría HCSBA. Mayo-agosto 1999

\begin{tabular}{|c|c|c|c|c|}
\hline \multirow[t]{2}{*}{ Tipo de complicación } & \multirow{2}{*}{$\begin{array}{c}\text { Grupo 1* } \\
\left(\begin{array}{c}n=46) \\
\mathbf{N}^{\circ}\end{array}\right.\end{array}$} & \multirow{2}{*}{$\begin{array}{c}\text { Grupo 2* } \\
\left(\begin{array}{c}(\mathrm{n}=129) \\
\mathbf{N}^{\circ}\end{array}\right.\end{array}$} & \multicolumn{2}{|c|}{ Total } \\
\hline & & & $\mathbf{N}^{\circ}$ & $\%$ \\
\hline Sobreinfección bacteriana & 13 & 28 & 41 & 23,4 \\
\hline Atelectasia & 13 & 16 & 29 & 16,5 \\
\hline Convulsión febril & 2 & 1 & 3 & 1,1 \\
\hline Otitis media aguda & - & 4 & 4 & 2,2 \\
\hline Influenza como IIH** & 12 & 8 & 20 & 11,4 \\
\hline Ingreso a UCIP $*$ & 8 & 4 & 12 & 6,8 \\
\hline Ventilación mecánica & 5 & 2 & 7 & 4 \\
\hline Fallecidos & 2 & - & 2 & 1,1 \\
\hline
\end{tabular}

UCIP: Unidad Cuidados Intensivos Pediátricos; IIH: Infección Intrahospitalaria; * Ver texto; ** Estadísticamente significativo mediante prueba de diferencia de porcentajes $\mathrm{Z}$ score $=3,7$ lo que representa un alfa $<0,05$.

La adquisición nosocomial de influenza fue de $11,4 \%$, correspondiendo a 20 pacientes, $12 \mathrm{de}$ los cuales pertenecían al Grupo 1 y 8 al Grupo 2. $\mathrm{Al}$ evaluar estos resultados por medio de la comparación de porcentajes (z score) se encontró un error alfa $<0,05$ lo que demuestra una diferencia estadísticamente significativa entre los grupos.

La oxigenoterapia se prolongó durante $9,3 \pm 12$ días en los pacientes del Grupo 1 y por $4,15 \pm 4,12$ días en el Grupo 2, resultando en un alfa $<0,05$. Se observó además que los pacientes del Grupo 1 prolongaban su tiempo de hospitalización (13,6 \pm 14,6 días) en comparación al Grupo 2 (5,9 \pm 4,7 días), $\mathrm{p}<0,05$.

Dos pacientes hospitalizados recibieron vacuna contra influenza para esta temporada, 1 en cada grupo de estudio; sin embargo, sólo recibieron la mitad de la dosis recomendada para su edad.

Dos pacientes fallecieron, pertenecientes al Grupo 1, ambos tenían deterioro neurológico. En uno de ellos existía el antecedente de obstrucción de la vía aérea por estenosis subglótica adquirida y durante su influenza presentó una sobreinfección, evolucionando con síndrome de distress respiratorio agudo. El segundo paciente cursó con una sobreinfección bacteriana sospechada por el cambio en su patrón radiológico y viraje en los parámetros de laboratorio, sin aislamiento bacteriológico, que lo llevó a falla multiorgánica y subsecuentemente a la muerte. La tasa de letalidad de influenza en este período alcanzó a 1,14\%.

Ocho pacientes presentaron una reinfección por FluA durante este período, con igual número de rehospitalizaciones, en 7 de ellos se obtuvo IFD positiva en dos ocasiones en diferentes pe- ríodos durante el tiempo de seguimiento y en otro, en tres oportunidades. Seis pertenecían al Grupo 1.

\section{Discusión}

Aunque la influenza no tiene elementos clínicos específicos, su aparición en la comunidad y el aumento del número de hospitalizaciones por su causa sigue claramente un patrón cíclico estacional, que se repite anualmente. En este estudio, la duración total de hospitalizaciones por influenza en niños fue mayor que la habitual (14 semanas), incluso mayor a la reportada por Chile a OMS en ese año (4 semanas) y que sólo corresponde al período de mayor incidencia (semanas 18 a 22$)^{17}$.

La sospecha clínica de la influenza se puede confirmar por métodos de diagnóstico sencillos en los que el método ideal es el cultivo viral, pero que podría ser superada por métodos más sensibles y específicos como la identificación del material genético viral por RPC y secuenciación, que sólo se realiza en el centro colaborativo de influenza dependiente de la OMS (CDC, Atlanta, E.U.A.) y evidentemente implica un mayor costo ${ }^{18}$. En nuestro país y en el HCSBA, se cuenta con IFD para la detección de antígeno viral, que tiene una sensibilidad estimada en alrededor de $75 \%$ y que puede llegar a $90 \%$ en período pico de la epidemia lo que valida el método de diagnóstico empleado en los pacientes evaluados.

Fue claro el predominio de casos causados por FluA en comparación a FluB y, aunque para el análisis de este estudio se eligió sólo a pacientes 
con IFD positiva a un virus, hubo un número importante de pacientes que presentaron coinfección viral, dándose con mayor frecuencia la asociación FluA con VRS, fenómeno reportado y que en epidemias de influenza puede alcanzar a $77 \%$ de los pacientes ${ }^{19-20}$. Si bien es cierto, clínicamente no se puede inferir cuál virus es causante de la enfermedad y cuál no, este hallazgo pone en duda el conocido concepto de interferencia viral, en que un virus ocupa determinado tejido impidiendo que otro lo haga en forma simultánea.

Los hallazgos clínicos en el grupo de pacientes estudiados fueron similares a los reportados por otros autores, teniendo como principal motivo de consulta la fiebre alta, generalmente superior a $38,5^{\circ} \mathrm{C}$, acompañada de otros signos poco específicos. La influenza evolucionó en forma autolimitada pero en el selecto grupo de pacientes hospitalizados, y por tanto de mayor gravedad que de los existentes en la comunidad, nuestros hallazgos apuntan a que aproximadamente 50\% podría presentar algún tipo de complicación, independientemente de si el paciente tenía patología crónica o no.

La efectividad de la vacuna dependerá de si la cepa viral circulante en el período epidémico está incluida en su formulación. En 1999, según los datos proporcionados por los centros de vigilancia ubicados en Santiago, la cepa viral circulante fue A/Sydney/5/97 (H3N2) ${ }^{18}$, presente en la vacuna recomendada para el período de invierno de ese año y que además contenía las cepas A/ Beijing/262/95 (H1N1) y la B/Beijing 184/9321. Aunque cada año se dispone de la vacuna apropiada y conocemos sus indicaciones precisas, nuestros resultados revelan que la población pediátrica de riesgo conocido no recibió vacunación o si lo hizo, no recibió la dosificación adecuada. Lo anterior sugiere que, aunque existe una campaña pública nacional de vacunación implementada anualmente, un grupo de pacientes de riesgo no son incluidos en ella, debiendo entonces efectuarse mayores esfuerzos en el futuro para la cobertura de estos niños. Mención aparte merecen los 56 lactantes bajo 6 meses de edad, en quienes la vacunación contra influenza no es recomendada por su escasa respuesta inmune, y para quienes las medidas preventivas debieran apuntar a los adultos o contactos que conviven con ellos.

Aunque el porcentaje de complicaciones no difiere entre ambos grupos, preocupa el grupo de pacientes crónicos, quienes al complicarse prolongaron el tiempo de estadía hospitalaria, el uso de oxigenoterapia, requirieron derivación a UCI o adquirieron nuevas infecciones una vez interna- dos. Las variables analizadas demostraron una diferencia estadísticamente significativa y de enorme importancia clínica en lo que respecta a la planificación de medidas de control futuras distintas de la vacunación.

Llamó la atención la adquisición de la enfermedad por contagio intrahospitalario así como de cuadros diarreicos por rotavirus. Debemos aclarar que los casos de influenza nosocomial no correspondieron en rigor a un brote, lo anterior debido a lo espaciado de su aparición en el tiempo y por casos índices en distintos sectores del Servicio de Pediatría. Aunque para influenza la recomendación emanada del CDC y basada en el mecanismo de transmisión, sugiere precauciones de gotitas y contacto ${ }^{22}$, en países como el nuestro y al igual que con infecciones por ADV, los pacientes con sospecha de influenza debieran ser aislados de preferencia en salas individuales o en cohortes de pacientes que compartan el mismo agente etiológico. Otras importantes medidas de implementación y que requieren supervisión permanente incluyen el lavado de manos, uso de mascarilla y cuidados específicos de superficies y objetos inanimados ${ }^{23}$. Finalmente y en relación a este tópico, debemos fomentar y aumentar la cobertura de vacunación contra influenza en el equipo de salud, excluir de la atención directa de pacientes al personal con infecciones respiratorias virales agudas, evitar el hacinamiento y controlar el número y estado de salud de las visitas. El conjunto de estas medidas, llevadas a la práctica por todo el equipo de salud, no tiene otro fin que evitar infecciones intrahospitalarias, disminuir costos económicos y sociales, mejorando la calidad de la atención.

Las herramientas terapéuticas de reciente incorporación, oseltamivir y zanamivir, la mejoría en la calidad y cobertura de vacunación, en conjunto con permanentes políticas de salud pública y hospitalaria, permitirían un mejor control de este agente de elevada morbilidad.

\section{Resumen}

La influenza se presenta como brotes estacionales y afecta con mayor severidad a la población con factores de riesgo conocidos. Se presenta, mediante estudio prospectivo, el comportamiento clínico epidemiológico de la influenza en niños hospitalizados durante el brote de 1999 en el Hospital Clínico San Borja Arriarán, comparando complicaciones en pacientes con y sin factores de riesgo. El diagnóstico se realizó por IFD. Se evaluó a 175 niños, 95\% con influenza A, 
$60 \%$ bajo 12 meses de edad. El Grupo 1 incluyó 46 niños con factores de riesgo y el Grupo 2 a 129 niños sin morbilidad previa. Hubo complicaciones en $51 \%$. En el Grupo 1 se encontró diferencia estadísticamente significativa en relación a adquisición nosocomial y estadía en unidad de cuidados intensivos pediátrica, además de promedios en días de oxigenoterapia y estadía hospitalaria $(\mathrm{p}<0,05)$. Se reconocen niños con mayor riesgo, en quienes deben focalizarse medidas preventivas como vacuna y programas de control de infecciones intrahospitalarias entre otras.

\section{Bibliografía}

1.- CDC Prevention and control of Influenza. Recommendations of the Advisory Committee on Immunization Practices (ACIP). Morb Mortal Wkly Rep MMWR 1999; 48 ( No. RR-4).

2.- Glezen W. Emerging infections: Pandemic influenza. Epidemiol Rev 1996; 18: 1-13.

3.- Treanor J J. Influenza virus. In Mandell Douglas and Bennett's Principles and Practice of Infectious Diseases. Mandell GL, Bennett JE, Dolin R (eds): $5^{\text {th }}$ ed. New York, Churchill Livingstone, 2000, p 1823.

4.- Simonsen L, Schonberger L, Stroup D, Arden N, Cox N. The impact of influenza on mortality in the USA. In Brown LE, Hampson AW, Webster RG, eds. Options for the control of influenza III. Amsterdam: Elsevier Science BV 1996: 26-33.

5.- Lui K-J, Kendal A. Impact of influenza on mortality in the United States from October 1972 to May 1985. Am J Public Health 1987; 77: 712-6.

6.- Serwint J, Miller R. Why diagnose influenza infections in hospitalized pediatric patients. Pediatr Infect Dis J 1993; 12: 200-4.

7.- Hall C. Nosocomial viral respiratory infections: perennial weeds on pediatric wards. Am J Med 1981; 70: 670-6.

8.- Muñoz F M, Campbell J, Atmar R et al. Influenza A virus outbreak in neonatal intensive care unit. Pediatr Infect Dis J 1999; 18: 811-5.
9.- Fox T, Cooney M, Hall C, Foy H. Influenza virus infections in Seattle families, 1975-1979. II. Pattern of infection in invaded households and relation of age and prior antibody to occurrence of infection and related illness. Am J Epidemiol 1982; 116: 228-42.

10.- Barnett E. Influenza immunization for children. $\mathrm{N}$ Engl J Med 1998, 338; 20: 1459-61.

11.- Carrat F, Tachet A, Rouzioux C, Housset B, Valleron A-J. Evaluation of clinical case definitions of Influenza: Detailed investigation of patients during the 19951996 epidemic in France. Clin Infect Dis 1999; 28: 283-90.

12.- Simonsen L, Fukuda K, Lawrence B, Schonberger, Cox N. The impact of Influenza epidemics on hospitalizations. J Infect Dis 2000; 181: 831-7.

13.- Cox N, Brammer L, Regnery H. Global influenza surveillance for pandemic or epidemics variants. Eur $\mathbf{J}$ Epidemiol 1994; 10: 467-70.

14.- Belshe R, Meldeman P, Treanor J et al. The efficacy of live attenuated, cold adapted, trivalent, intranasal influenza virus vaccine in children. $\mathrm{N}$ Engl $\mathrm{J}$ Med 1998; 338: 1405-12.

15.- Clements D, Langdon L, Bland C, Walter E. Influenza A vaccine decreases the incidence of otitis media in 6-30 month-old children in day care. Arch Pediatr Adolesc Med 1995; 149: 1113-7.

16.- Delpiano L, Guillén B, Casado M C. Influenza en pediatría. Brotes 1998-1999. Libro de Resúmenes XVI Congreso Chileno de Infectología 1999, CO - 56.

17.- Flunet WHO http://www.who.int/emc/diseases/flu/ recommendations.html

18.- Storch G, Diagnostic virology. Clin Infect Dis 2000; 31: 739-51.

19.- Tsai H, Kuo P, Liu C, Wang J. Respiratory viral infections among pediatric inpatients and outpatients in Taiwan from 1997 to 1999. J Clin Microbiol 2001; 39: $111-8$

20.- Subi K. Mixed respiratory viral infections in Estonia: a long-term laboratory study. Acta Virol 1998; 42: 413-5.

21.- Weekly Epidemiological Record 1999; 73 (40).

22.- Edmond M. Isolation. Infect Control Hosp Epidemiol 1997; 18: 58-64.

23.- Normas para la prevención de transmisión asociada a la atención en salud, de agentes etiológicos virales de infecciones respiratorias agudas en pacientes pediátricos. Circular 4C/18 MINSAL, Mayo 1999.

Correspondencia a:

Luis Delpiano Méndez

E-mail: ludelpia@mi.terra.cl 